\title{
Two-fluid models of cosmic-ray modified radiative shocks including the effects of an acoustic instability
}

\author{
A. Y. Wagner ${ }^{1}$, S. A. E. G. Falle ${ }^{2}$, and T. W. Hartquist ${ }^{1}$ \\ ${ }^{1}$ School of Physics and Astronomy, University of Leeds LS2 9JT, UK \\ e-mail: ayw@ast.leeds.ac.uk \\ 2 Department of Applied Mathematics, University of Leeds, Leeds LS2 9JT, UK \\ Received 28 August 2006 / Accepted 27 October 2006
}

\section{ABSTRACT}

\begin{abstract}
Aims. We extend the study of two-fluid models of cosmic-ray modified radiative shocks by including energy transfer from the cosmic rays to the thermal gas due to a cosmic-ray driven acoustic instability.

Methods. We perform hydrodynamic simulations to find solutions to the time-dependent, one-dimensional two-fluid equations governing the thermal fluid and cosmic-ray fluid. Source terms transferring energy between the two fluids are included.

Results. The magnitudes of the source terms determine the degree to which the cosmic rays modify the radiative shock. We found a range of steady solutions characterised by moderate cosmic-ray acceleration and a compression ratio significantly greater than 7 , which is the limit for cosmic-ray dominated shocks. The post-shock temperature and overall compression increase as the source term in the thermal fluid's energy equation increases. For sufficiently strong coupling, the solutions are thermally overstable.
\end{abstract}

Key words. shock waves - ISM: cosmic rays - hydrodynamics - instabilities - ISM: supernova remnants

\section{Introduction}

Wagner et al. (2006) have performed one-dimensional two-fluid simulations of cosmic-ray modified radiative shocks. They have shown that cosmic rays suppress the thermal overstability of radiative shocks. Steady solutions were found for all Mach numbers greater than 3 . The cosmic ray acceleration in these steady solutions is very efficient and the post-shock flow is cosmic-ray dominated. The overall compression ratio is, therefore, always limited to 7 or less. If the diffusion length is much larger than the cooling length the shock is nearly isothermal.

Observations of supernova remnants such as the Cygnus Loop (Raymond et al. 2001) imply shock compression ratios much greater than 7 and substantial heating of the thermal gas. To reconcile observations with radiative shock models that include cosmic ray acceleration, we explore an additional coupling between the cosmic ray fluid and thermal fluid that transfers energy from the former to the latter. This will limit the acceleration of the cosmic rays and enhance the compression of the thermal fluid.

Völk et al. (1984) have investigated the dissipation of Alfvén waves generated by cosmic ray streaming, which transfers energy from the cosmic rays to the thermal fluid. However, they have found that the dissipation is not efficient at high Alfvénic Mach numbers.

Drury \& Falle (1986) have studied a cosmic-ray driven acoustic instability that amplifies density perturbations in regions where the cosmic ray gradient is large. They have determined the condition for instability through a linear perturbation analysis and verified their results with simulations. In regions where the instability occurs, a net energy transfer from the cosmic-ray fluid to the thermal fluid occurs.

In this work we have constructed models of cosmic-ray modified radiative shocks which couple the cosmic ray fluid to the thermal fluid through source terms that act in a way that tends to drive the medium to a state that is stable to the Drury \& Falle (1986) type instability. We first introduce the two-fluid equations with the coupling terms and relevant parameters in Sect. 2. In Sect. 3 we will show a series of steady solutions as well as solutions that are thermally overstable (e.g. Pittard et al. 2005). We close the paper with concluding remarks in Sect. 4

\section{Two-fluid equations}

The following one-dimensional hydrodynamic equations govern the thermal fluid and the cosmic ray fluid:

$\frac{\partial \rho}{\partial t}+\frac{\partial \rho u}{\partial x}=0$

$\frac{\partial \rho u}{\partial t}+\frac{\partial \rho u^{2}}{\partial x}+\frac{\partial P_{\mathrm{G}}}{\partial x}+\frac{\partial P_{\mathrm{C}}}{\partial x}=0$,

$\frac{\partial}{\partial t}\left(\frac{\rho u^{2}}{2}+\frac{P_{\mathrm{G}}}{\gamma_{\mathrm{G}}-1}\right)+\frac{\partial}{\partial x}$
$\left(\frac{\rho u^{3}}{2}+\frac{\gamma_{\mathrm{G}} P_{\mathrm{G}} u}{\gamma_{\mathrm{G}}-1}+P_{\mathrm{C}} u\right)$
$-P_{\mathrm{C}} \frac{\partial u}{\partial x}+\rho^{2} \mathcal{L}(T)=S_{\mathrm{G}}$,

$\frac{\partial P_{\mathrm{C}}}{\partial t}+\frac{\partial P_{\mathrm{C}} u}{\partial x}+\left(\gamma_{\mathrm{C}}-1\right) P_{\mathrm{C}} \frac{\partial u}{\partial x}-\kappa \frac{\partial^{2} P_{\mathrm{C}}}{\partial x^{2}}=S_{\mathrm{C}}$.

$\rho, P_{\mathrm{G}}, T$, and $\gamma_{\mathrm{G}}$ are the density, pressure, and temperature, and adiabatic index of the thermal gas, respectively. $P_{\mathrm{C}}, \kappa$, and $\gamma_{\mathrm{C}}$ are the cosmic ray pressure, the cosmic ray diffusion coefficient and the cosmic ray adiabatic index. $x$ and $t$ are the space and time coordinates, and $u$ is the speed of the flow. We assume $\gamma_{\mathrm{G}}$, and $\gamma_{\mathrm{C}}$ to be constant with $\gamma_{\mathrm{G}}=5 / 3$ and $\gamma_{\mathrm{C}}=4 / 3$.

Except very near to the upstream temperature, the cooling rate per unit volume, $\rho^{2} \mathcal{L}(T)$, is taken to be

$\rho^{2} \mathcal{L}(T)=\rho^{2} \Lambda T^{\alpha}$, 
where $\Lambda$ and $\alpha$ are constants. The gas is allowed to cool to the upstream temperature, towards which $\mathcal{L} \rightarrow 0$. $\mathcal{L}<0$ if $T$ is less than the upstream temperature. Apart from the source terms $S_{\mathrm{G}}$ and $S_{\mathrm{C}}$, Eqs. (1)-(4) are the same equations as those used by Wagner et al. (2006).

If $\kappa$ is independent of time and space, the condition for instability of the type described by Drury \& Falle (1986) can be written

$\frac{\kappa}{a}>\gamma_{\mathrm{C}} P_{\mathrm{C}}\left|\frac{\partial P_{\mathrm{C}}}{\partial x}\right|^{-1}$,

where $a=\sqrt{\gamma_{\mathrm{G}} P_{\mathrm{G}} / \rho}$ is the adiabatic speed of sound. Condition (6) is relevant at any point in the flow, except at the gas subshock, if one exists. The density perturbations, thus, grow if the lengthscale associated with the cosmic rays, $P_{\mathrm{C}} /\left|\partial P_{\mathrm{C}} / \partial x\right|$, is less than a critical lengthscale of order $\kappa / a$. In cosmic-ray modified shocks, the precursor lengthscale is of the order of the diffusion length

$l_{\mathrm{d}}=\frac{4 \kappa}{u_{\mathrm{s}}}$,

where $u_{\mathrm{s}}$ is the shock speed. Thus, the criterion for instability is always satisfied in the precursor of strong shocks, since $u_{\mathrm{s}} \gg a$.

The source terms in Eqs. (3) and (4) are

$S_{\mathrm{C}}=\left\{\begin{aligned} &-\frac{\gamma_{\mathrm{C}}-1}{\gamma_{\mathrm{G}}-1} P_{\mathrm{G}}\left(\frac{\kappa}{a}\left|\frac{\partial P_{\mathrm{C}}}{\partial x}\right| \frac{1}{\gamma_{\mathrm{C}} P_{\mathrm{C}}}-1\right) \frac{1}{\tau} \\ & 0 \text { if } \frac{\kappa}{a}\left|\frac{\partial P_{\mathrm{C}}}{\partial x}\right| \frac{1}{\gamma_{\mathrm{C}} P_{\mathrm{C}}}-1>0 \\ & 0 \text { if otherwise }\end{aligned}\right.$

$S_{\mathrm{G}}=-\frac{S_{\mathrm{C}}}{\gamma_{\mathrm{C}}-1}$.

$\tau$ is a time constant that characterizes the energy transfer rate.

$S_{\mathrm{C}}$ is proportional to the difference between the local state of the fluid and the state of critical stability given by (6). The magnitude of the source term, thus, increases linearly with the cosmic ray pressure gradient. The effect of the source terms is to drive the flow towards critical stability. The total energy is conserved in this process.

We note that the instability is inherent in the Eqs. (1)-(4). By including the source terms we are bypassing the initial perturbations and assuming the instability to be sufficiently strong to achieve the desired energy transfer rate.

We use the same dimensionless parameters as Wagner et al. (2006) to specify the upstream boundary conditions and flow parameters of solutions: the upstream Mach number with respect to the thermal sound speed

$M_{0}=\sqrt{\frac{u_{0}^{2} \rho_{0}}{\gamma_{\mathrm{G}} P_{\mathrm{G} 0}}} ;$

the exponent $\alpha$ in the cooling law; and the ratio of the cosmic ray diffusion length, $l_{\mathrm{d}}$, to the cooling length, $l_{\mathrm{c}}$,

$q=\frac{l_{\mathrm{d}}}{l_{\mathrm{c}}}$

Here

$l_{\mathrm{c}}=\frac{u_{\mathrm{s}} \mu k T_{\mathrm{s}}}{\rho \Lambda_{0} T_{\mathrm{s}}^{\alpha}}$,

where $\rho_{\mathrm{s}}$ and $T_{\mathrm{s}}$ are the immediate post-shock density and temperature given by the Rankine-Hugoniot jump conditions for a single-fluid thermal medium. $\mu$ and $k$ are the average mass per gas particle and Boltzmann's constant. Throughout the paper, the subscript 0 denotes asymptotic upstream values. For a steady shock, $u_{\mathrm{s}}=u_{0}$, and we will assume the equality when calculating the diffusion and other lengthscales associated with a shock.

We choose the ratio of the coupling lengthscale, $l_{\mathrm{e}}=\tau u_{0}$, to the diffusion length to be $\eta$, to quantify the strength of the coupling:

$\eta=\frac{l_{\mathrm{e}}}{l_{\mathrm{d}}}=\frac{\tau u_{0}^{2}}{4 \kappa}$.

To limit parameter space we will restrict ourselves to cases where $\alpha=-0.5$ (see e.g. Kahn 1976) and $M_{0}=10$ and $M_{0}=5$. Because the cosmic ray pressure and thermal pressure are comparable in the interstellar medium (e.g. Ferrière 1998), we set $\left(\gamma_{\mathrm{C} 0} P_{\mathrm{C} 0}\right) /\left(\gamma_{\mathrm{G} 0} P_{\mathrm{G} 0}\right)=1$. We will consider cases where $q=1$ and $q=10$. Our chosen set of values, thus, represents a portion of parameter space that is relevant for radiative supernova remnant shocks.

Equations (1)-(4) are solved numerically with the same method as Wagner et al. (2006) employed.

\section{Results}

We first present examples of steady solutions and thermally overstable solutions to Eqs. (1)-(4). In all figures for steady solutions, the flow enters the grid from the left. Free flowing boundary conditions are applied on either side of the grid. For thermally stable solutions, the steady state is found when the downstream gradients vanish. The solutions are shown in the frame of the shock. For thermally overstable solutions, the solutions are shown in the frame of the mean shock position. The downstream flow fluctuates about a time-independent average.

In general, one can identify four regions of the flow: the asymptotic upstream region, the cosmic ray precursor bounded by the gas subshock; the cooling region between the gas subshock and the cold dense layer, and the asymptotic downstream region. If substantial cosmic ray acceleration occurs in the precursor region, the gas subshock is smoothed out. As expected, we have found the source terms to be non-zero in the cosmic-ray precursor region of $M_{0}=10$ and $M_{0}=5$ shocks. Condition (6) is never found to be satisfied in the cooling region. We note that the precursor region of $M_{0}=3$ shocks for both $q=1$ and $q=10$ is stable and the source terms vanish. The instability is, thus, only important in reasonably strong cosmic-ray modified radiative shocks.

We have found several examples where there exist more than one solution for a given set of upstream conditions and flow parameters. Such non-linear solution spaces have been identified in two-fluid models of adiabatic cosmic-ray modified shocks (Drury \& Völk 1981; Becker \& Kazanas 2001). Here, the existence of multiple solutions depends also on $\eta$. For $M_{0}=10, q=$ 10 shocks we have found two solutions when $0.50 \lessgtr \eta \lesssim 0.55$. If $M_{0}=10, q=1$, there are two solutions when $\eta \lesssim 0.5$. For $M_{0}=5, q=10$ and $q=1$ shocks, we have not found values for $\eta$ for which multiple solutions occur.

One of the solutions can always be found by applying a reflective boundary at one end of the grid. After a well defined shock structure builds up and moves away from the reflective boundary, we transform to the frame of the shock and change the reflective boundary to a free flowing boundary. Thus, for a given $M_{0}$ and $q$, we first find one solution for each of a series of values of $\eta$. An abrupt change in the jump conditions between two solutions for proximate values of $\eta, \eta_{\mathrm{a}}$ and $\eta_{\mathrm{b}}$, where 

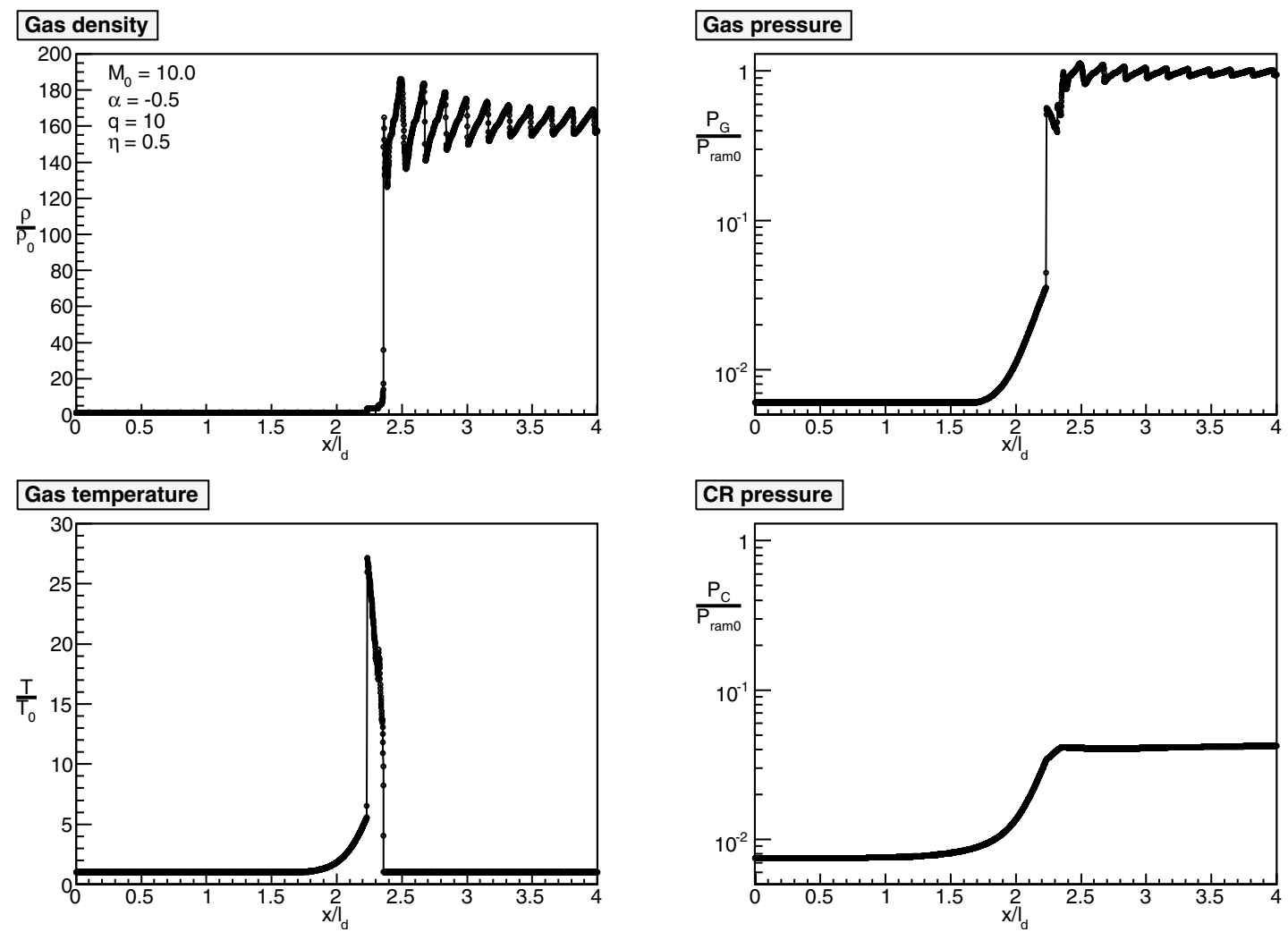

Fig. 1. Density, pressure, temperature, and cosmic ray pressure profiles of a $M_{0}=10, q=10$, and $\eta=0.5$ shock. The density and temperature are given in units of their upstream values. The gas pressure and cosmic ray pressure are given in units of the upstream ram pressure. The abscissa is in units of the diffusion length. This solution is thermally overstable. The overall compression ratio is close to $\gamma_{\mathrm{G}} M_{0}^{2}$ and the maximum temperature is 28 times the upstream temperature. The cosmic pressure increases by a factor of 6 . There are two solutions with these upstream conditions and flow parameters. The other solution is shown in Fig. 2.

$\eta_{\mathrm{b}}>\eta_{\mathrm{a}}$ suggests the existence of multiple solutions in the vicinity of $\eta_{\mathrm{a}}$ or $\eta_{\mathrm{b}}$. To find these solutions and determine the domain in $\eta$ for which multiple solutions occur, we start with the solution for $\eta_{\mathrm{a}}$ and increase $\eta$ in small steps towards $\eta_{\mathrm{b}}$, and beyond if necessary. Likewise, we start with the solution for $\eta_{\mathrm{b}}$ and reduce $\eta$ in small steps towards $\eta_{\mathrm{a}}$, and below if necessary. We thereby explore a region of the $\eta$ domain, that may contain multiple solutions, through small incrementations of $\eta$ in both directions. We proceed with increasing and decreasing $\eta$ until the upper and lower limits of $\eta$ for which two solutions occur are reached, respectively. As in the case of adiabatic cosmic-ray modified shocks, the domains that we have mentioned, in which two solutions occur, may in fact contain a third, intermediate solution, which is likely to be unstable (Mond \& Drury 1998).

The four panels of Fig. 1 show the gas density, temperature, pressure, and cosmic ray pressure profiles of a $M_{0}=10, q=$ $10, \eta=0.5$ shock. The coupling between the cosmic rays and the thermal gas due to the acoustic instability is strong. Energy is transferred efficiently from the cosmic rays to the thermal gas and further cooling leads to an overall compression ratio close to $\gamma_{\mathrm{G}} M_{0}^{2}$. The maximum post-shock temperature occurs immediately behind the gas subshock and is 28 times the upstream temperature. The shock is thermally overstable, much like a radiative $M_{0}=10, \alpha=-0.5$ shock that is not modified by cosmic rays: the shock front oscillates in position with an amplitude of the order of the cooling length and the post-shock flow fluctuates strongly. The small increase in cosmic ray pressure by a factor of 6 is insufficient to suppress the thermal overstability.

The solution shown in Fig. 2, however, is subject to the same upstream conditions and the same values of $q$ and $\eta$ as the solution shown in Fig. 1. This solution is thermally stable. The compression ratio and maximum temperature are substantially smaller and most of the upstream kinetic energy goes into the cosmic rays.

Above $\eta \approx 0.55$ the unique solutions of $M_{0}=10, q=10$ shocks resemble the $M_{0}=10, q=10, \eta=0.5$ solution with the lower compression ratio (Fig. 2) and, with increasing $\eta$, they approach the limit of a cosmic-ray modified radiative shock with $S_{\mathrm{C}}=S_{\mathrm{G}}=0$. Below $\eta \approx 0.50$, the unique solutions resemble the solution with the higher compression ratio (Fig. 1) and approach the limit of a radiative shock in the absence of cosmic rays with decreasing $\eta$.

Figure 3 depicts a $M_{0}=10, q=1, \eta=0.5$ shock. These upstream conditions and parameters allow a second solution, which is shown in Fig. 4. The overstable shock shown in Fig. 3 differs from that shown in Fig. 1 only in its value of $q$. The lower value of $q$ here causes a slight drop in overall compression, yet a notably higher maximum post-shock temperature. Similar to the $M_{0}=10, q=10$ shocks, the second solution for $M_{0}=10, q=1, \eta=0.5$ (Fig. 4) exhibits a smaller overall compression and lower maximum temperature than the first solution. Compared to the shock in Fig. 2, where $q=10$, the maximum temperature is notably higher, while the compression ratios are similar. There is also a gas subshock, while in the case of $q=10$ the flow is smooth until the boundary to the cold dense layer. Comparing the pressure profiles of the shocks in Figs. 2 and 4, we note that, in the case where $q=1$, the gas pressure rises to a significant fraction of the upstream ram pressure before it decreases again and the cosmic ray fluid dominates the post-shock flow. The cosmic rays are only partially accelerated in the 

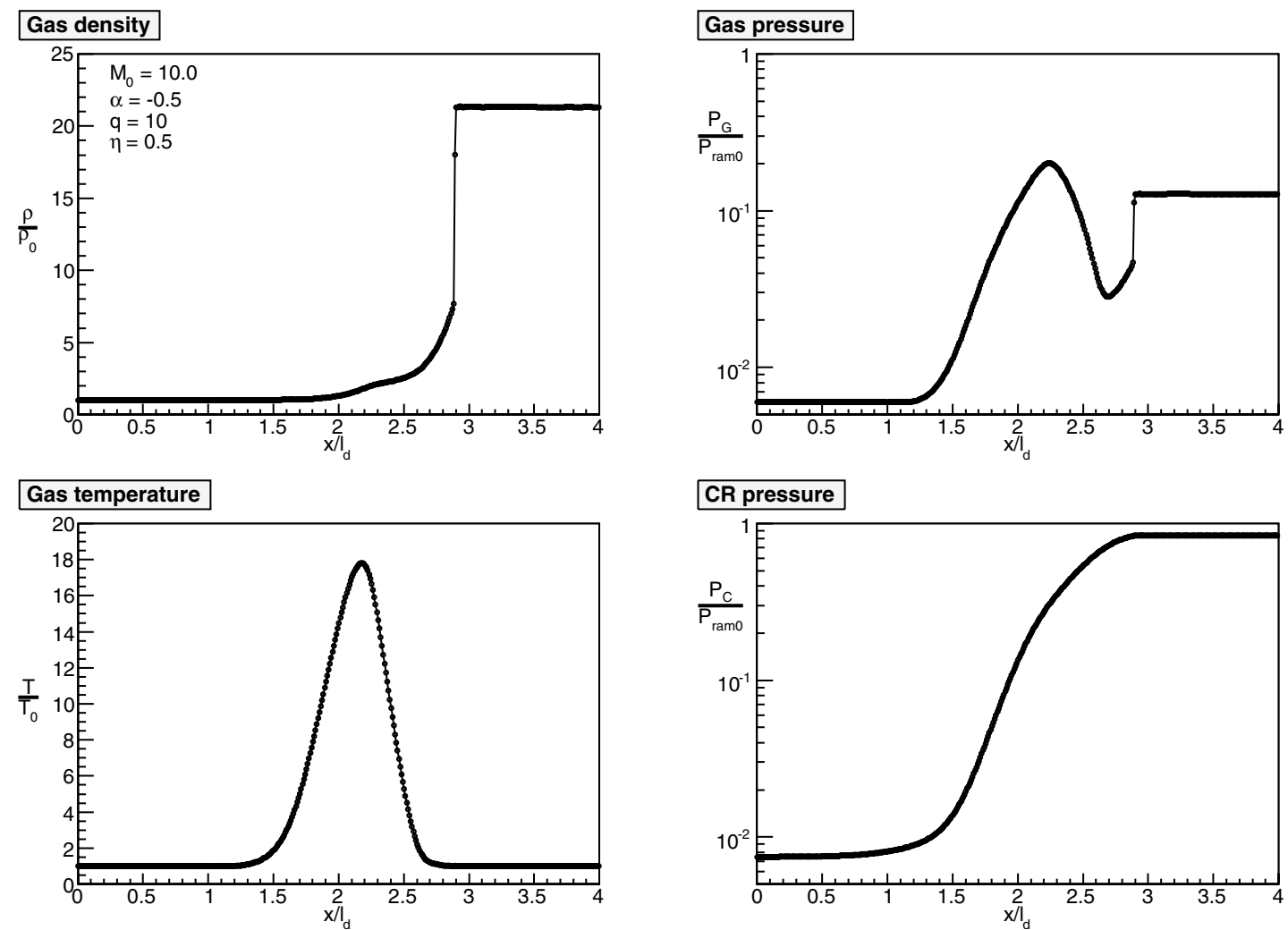

Fig. 2. Density, pressure, temperature, and cosmic ray pressure profiles of a $M_{0}=10, q=10$, and $\eta=0.5$ shock. The units are the same as in Fig. 1 . The overall compression ratio is about 21 and the solution is thermally stable. The temperature rises only to 18 times the upstream temperature. There are two solutions with these upstream conditions. The other is shown in Fig. 1.
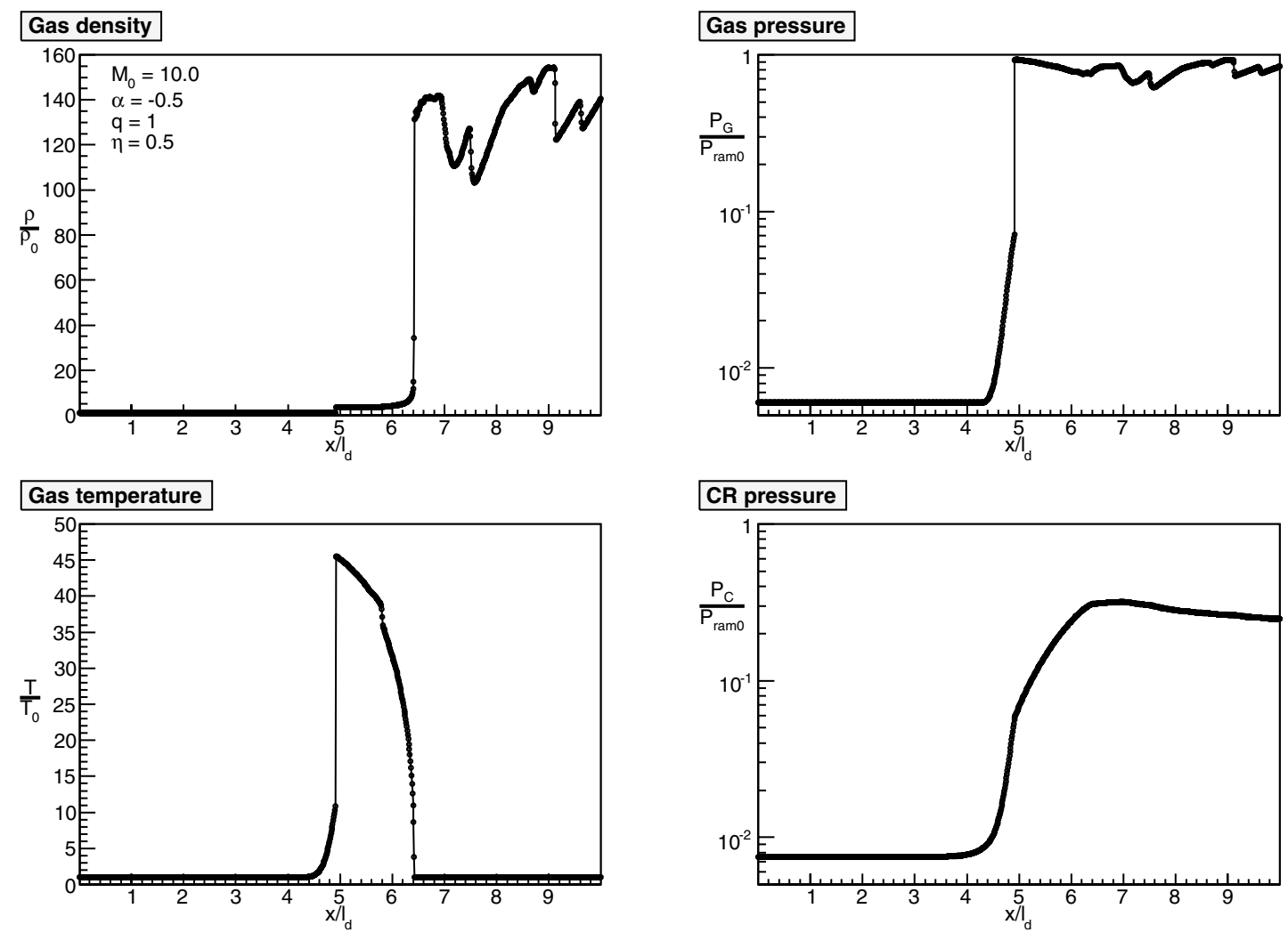

Fig. 3. Density, pressure, temperature, and cosmic ray pressure profiles of a $M_{0}=10, q=1$, and $\eta=0.5$ shock. The units are the same as in Fig. 1 . The overall compression ratio is about 135 , somewhat lower than $\gamma_{\mathrm{G}} M_{0}^{2}$. The solution is thermally overstable. The maximum temperature is about 45 times the upstream temperature. The cosmic ray pressure increases by a factor of 40 . There are two solutions with these upstream conditions. The other solution is shown in Fig. 4. 

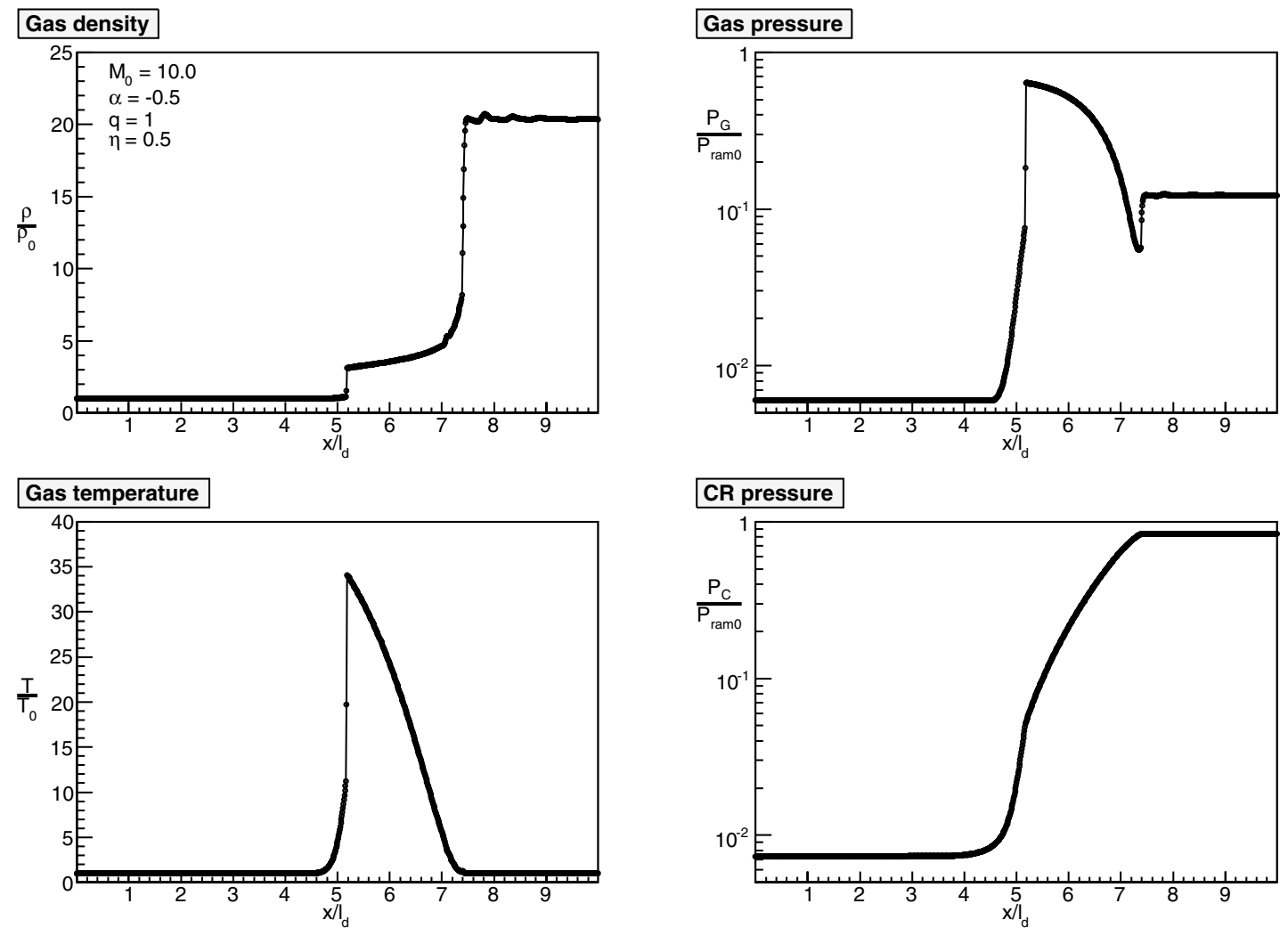

Fig. 4. Density, pressure, temperature, and cosmic ray pressure profiles. $M_{0}=10, q=1$, and $\eta=0.5$ shock. The units are the same as in Fig. 1 . The overall compression ratio is about 20 . The solution is thermally stable. The maximum temperature is about 34 times the upstream temperature. The cosmic ray pressure dominates the distant downstream flow. There are two solutions with these upstream conditions. The other solution is shown in Fig. 3.

precursor region and further accelerated in the cooling region. This allows for a greater rise in temperature and gas pressure in the precursor. In the cases where $q=10$, the cosmic ray acceleration in the precursor region is greater, reducing the jump in temperature. As in the case of cosmic-ray modified radiative shocks without the additional coupling, the overall compression ratio is only weakly sensitive to $q$.

Above $\eta \approx 0.5$, the unique solutions of $M_{0}=10, q=1$ shocks resemble the $M_{0}=10, q=1, \eta=0.5$ solution with the lower compression ratio (Fig. 4) and, with increasing $\eta$, they approach the limit of a cosmic-ray modified radiative shock with $S_{\mathrm{C}}=S_{\mathrm{G}}=0$.

Figure 5 shows a $M_{0}=5, q=1, \eta=0.03$ shock. Although the gas pressure dominates the immediate post-subshock region, the cosmic ray pressure and gas pressure are comparable in the distant downstream flow. The overall compression ratio is therefore only 13.3 and the shock is thermally stable. The compression ratio is not increased by lowering $\eta$.

A series of solutions for $M_{0}=5, q=10$ shocks for different values of $\eta$ is presented in the four panels of Fig. 6 . The value of $\eta$ is given next to each shock profile. "SR" denotes a radiative shock in the absence of cosmic rays and "SCR" denotes a cosmic-ray modified radiative shock with $S_{\mathrm{C}}=S_{\mathrm{G}}=0$. Smaller values of $\eta$ result in larger compression ratios, higher post-shock temperatures, larger asymptotic downstream gas pressure, and lower asymptotic downstream cosmic ray pressure. The thermal overstability occurs for sufficiently small $\eta$. Between $\eta \approx 0.1$ and $\eta \approx 0.3$ the solutions are more sensitive to $\eta$ than outside this interval. A value of $\eta$ of 0.15 is near the critical value for the suppression of thermal overstability. For $\eta=0.15$ the compression ratio is about 36 and the maximum temperature is about 8.5 times the upstream value.

Finally, we present two time-dependent runs following the procedure employed by Wagner et al. (2006). We start with a $M_{0}=5$ radiative shock without cosmic rays that is thermally overstable and introduce the cosmic rays homogeneously into the entire grid at time $t=0$. The initial homogeneous cosmic ray pressure is $P_{\mathrm{C}}=\gamma_{\mathrm{G}} / \gamma_{\mathrm{C}} P_{\mathrm{G} 0}$ and $q=10$. Free flowing boundary conditions are still used at either end of the grid. In the spacetime diagrams of the gas density and cosmic ray pressure (Figs. 7 and 8), the space coordinate is given in units of the diffusion length and the time coordinate is given in units of the diffusion time. The flow enters the grid from the bottom. The shading is logarithmic and darker shading denotes higher values. In the run with $\eta=0.3$ (see Fig. 7), the thermal overstability is damped as the cosmic rays are accelerated at the shock front and gain sufficient pressure downstream. A cosmic-ray modified shock similar to the shock with $\eta=0.3$ shown in Fig. 6 propagates away from the decaying cold dense layer. In the run with $\eta=0.1$ (see Fig. 8), the overstability is not damped as the cosmic rays are only weakly accelerated. The amplitude and period of the shock front oscillations, as well as the speed of the mean shock position, increase. The resulting shock is similar to the shock for $\eta=0.1$ (see Fig. 6).

\section{Conclusion}

We have found steady solutions as well as thermally overstable solutions of cosmic-ray modified radiative shocks including a coupling between the cosmic rays and the thermal gas that mimics the possible effects of an acoustic instability. The 

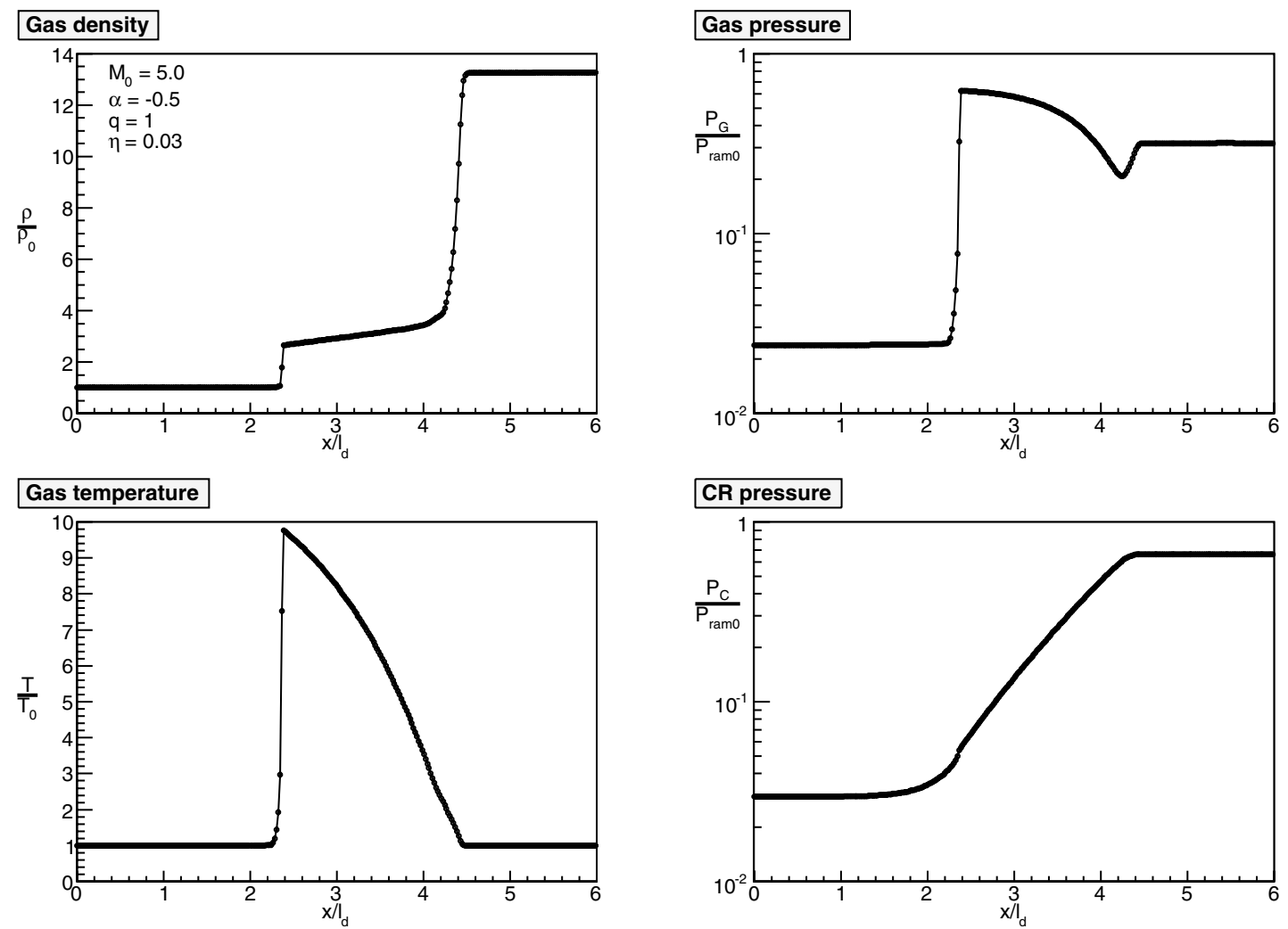

Fig. 5. Density, pressure, temperature, and cosmic ray pressure profiles for a $M_{0}=5, q=1$, and $\eta=0.03$ shock. The overall compression ratio is about 13.3 and the solution is thermally stable. The temperature rises to about 10 times the upstream temperature. The distant downstream cosmic ray pressure and gas pressure are comparable.
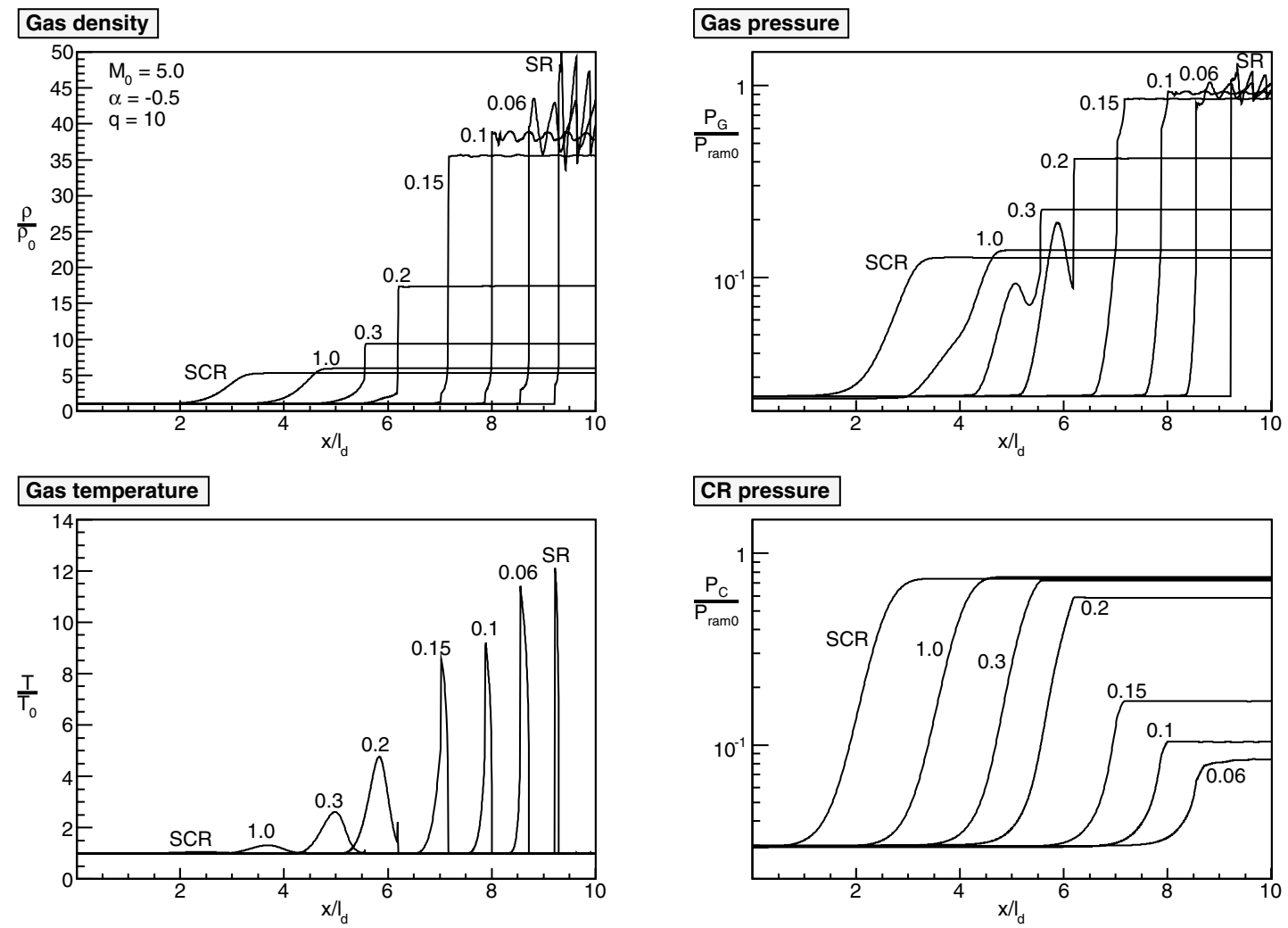

Fig. 6. The density, pressure, temperature, and cosmic ray pressure profiles in each panel represent a series of solutions for $M_{0}=5, q=10$ with varying $\eta$. The value of $\eta$ is indicated next to each profile. "SR" is a radiative shock in the absence of cosmic rays and "SCR" is a cosmic-ray modified radiative shock with $S_{\mathrm{C}}=S_{\mathrm{G}}=0$. The compression ratio, maximum temperature, and asymptotic downstream gas pressure increase with decreasing $\eta$. The asymptotic downstream cosmic ray pressure decreases with decreasing $\eta$. The thermal overstability occurs for sufficiently small $\eta$. 

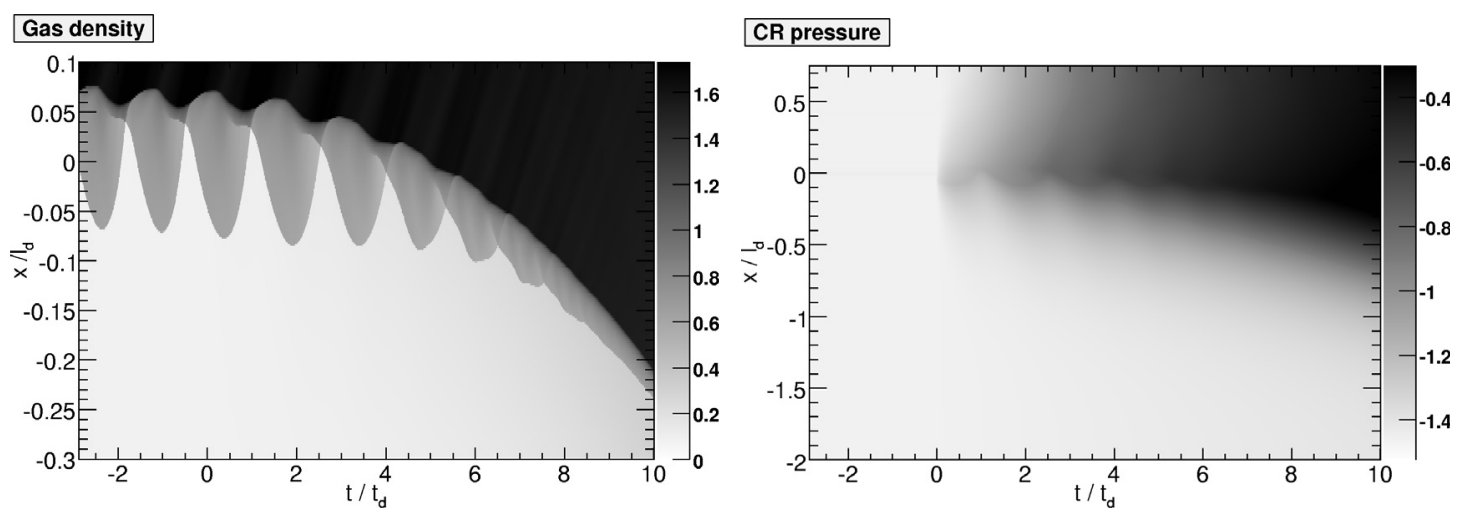

Fig. 7. Space-time diagram of the gas density and cosmic ray pressure. The abscissa is in units of the diffusion time, the ordinate in units of the diffusion length. The shading is logarithmic with darker shading representing higher values. At time $t<0$ the radiative $M_{0}=5$ shock does not include cosmic rays. At $t=0$ the cosmic rays are introduced homogeneously over the entire grid with pressure $P_{\mathrm{C}}=\gamma_{\mathrm{G}} / \gamma_{\mathrm{C}} P_{\mathrm{G} 0} . q=10$ and $\eta=0.3$. As the cosmic rays are accelerated at the shock front, the thermal overstability is suppressed.
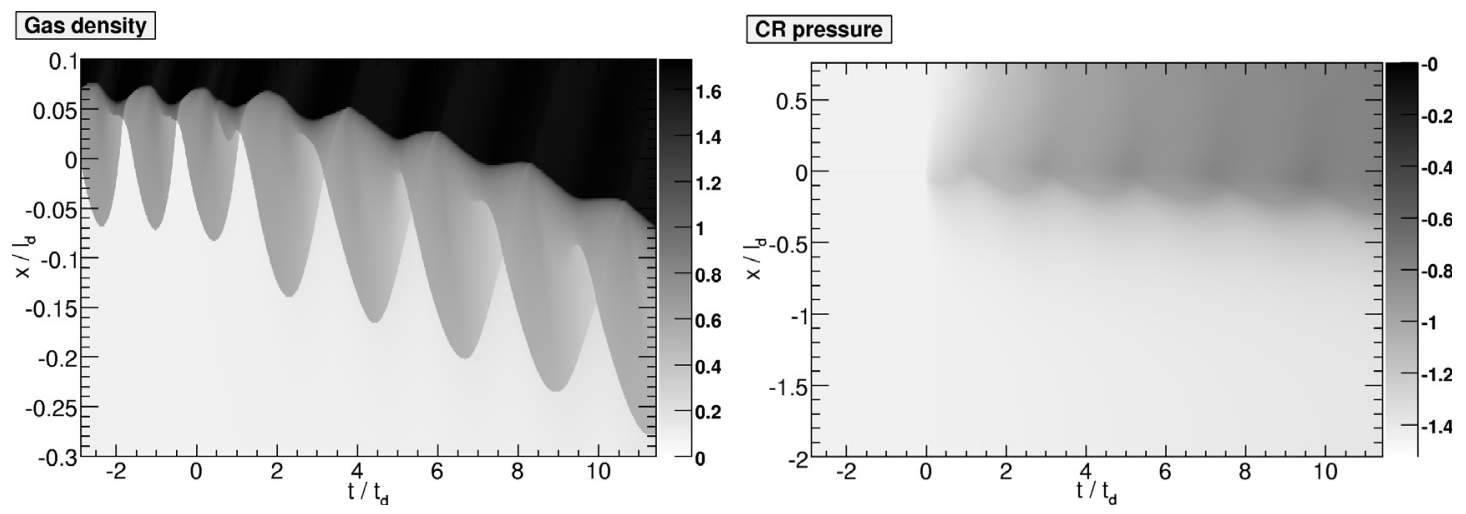

Fig. 8. Space-time diagram of the gas density and cosmic ray pressure. The initial conditions are the same as in Fig. 7. At $t=0$ the cosmic rays are introduced homogeneously over the entire grid with pressure $P_{\mathrm{C}}=\gamma_{\mathrm{G}} / \gamma_{\mathrm{C}} P_{\mathrm{G} 0} . q=10$ and $\eta=0.1$. The cosmic rays do not gain sufficient pressure to suppress the thermal overstability, which continues with a larger amplitude and longer period.

instability occurs in regions with a sufficiently steep cosmic ray gradient (Drury \& Falle 1986), such as in the precursor of strong cosmic-ray modified shocks. The instability effectively transfers energy from the cosmic rays to the thermal gas. The solutions range from shock structures that resemble cosmic-ray dominated radiative shocks to shock structures that resemble radiative shocks that are not modified by cosmic rays. These two cases are the limits between which the solutions that include the additional coupling between cosmic rays and thermal gas are found. The overall compression ratio and the maximum temperature increase when the coupling of the gas to the cosmic rays is stronger. For sufficiently strong coupling, the thermal overstability occurs. While the maximum temperature is also sensitive to the ratio of the diffusion length to the cooling length, the overall compression is not. In some regions of parameter space two solutions have been found. Cosmic-ray modified adiabatic shocks for which the coupling is neglected are known to have regions in parameter space with multiple solutions, whereas all solutions of cosmic-ray modified radiative shocks for which the coupling is neglected studied by Wagner et al. (2006) were found to be unique due to the isothermal asymptotic boundary conditions. The additional coupling contributes a degree of freedom between the cosmic-ray fluid and the thermal gas that reintroduces multiple solutions to the problem.

We have also presented results for two time-dependent runs. In the first case, where the additional coupling between cosmic rays and thermal gas was weaker, the downstream cosmic-ray pressure rose substantially and the thermal overstability was suppressed. In the other case, where the coupling was stronger and the gain in cosmic ray pressure less, the thermal overstability persisted with a larger amplitude and longer period.

Although we have shown that the acoustic instability can have a significant effect on the structure, our model for the source terms simply assumes that the instability tends to drive the system towards a marginally stable state. This is certainly reasonable, and has the advantage that it only contains one free parameter, but it has not been derived rigorously from first principles. Since such a derivation would be extremely difficult, it seems more sensible to adopt a semi-empirical approach to studying the dynamics of cosmic-ray modified shocks by using observations of spectral lines to infer the values $\rho_{0}, u_{\mathrm{s}}, \kappa$, and $\tau$. We will use this semi-emprical approach in future work.

\section{References}

Becker, P. A., \& Kazanas, D. 2001, ApJ, 546, 429

Drury, L. O’C., \& Völk, J. H. 1981, ApJ, 248, 344

Drury, L. O'C., \& Falle, S. A. E. G. 1986, MNRAS, 223, 353

Ferrière, K. 1998, ApJ, 497, 759

Kahn, F. D. 1976, A\&A, 50, 145

Mond, M., \& Drury, L. O'C. 1998, A\&A, 332, 385

Pittard, J. M., Dobson, M. S., Durisen, R. H., et al. 2005, A\&A, 438, 11

Raymond, J. C., Li, J., Blair, W. P., \& Cornett, R. H. 2001, ApJ, 560, 763

Völk, H. J., Drury, L. O'C., \& McKenzie, J. F. 1984, A\&A, 130, 19

Wagner, A. Y., Falle, S. A. E. G., Hartquist, T. W., \& Pittard, J. M. 2006, A\&A, 452,763 